\title{
"Experiencia de la escuela normal superior santa Ana de Baranoa en el diseño e implementacion de un sistema de Gestión Ambiental"
}

\section{Experience of escuela normal santa ana de baranoa in design and implementation of a manage enviromental system.}

Por: Silvera Rada, Ibón*

Resumen: El proyecto de investigación “Diseño e implementación de un Sistema de Gestión Ambiental en la Escuela Normal Superior Santa Ana y el barrio Santa Ana del municipio de Baranoa, que fomente la participación de la comunidad educativa en el manejo adecuado de los residuos sólidos" ; se viene realizando desde el año 2006 y actualmente se encuentra vigente.

A través de éste proyecto se buscó diseñar e implementar un Sistema de Gestión Ambiental en la Escuela y el barrio, que fomentara la participación de la comunidad educativa y de los habitantes del barrio, con el propósito de plantear alternativas de solución en torno a la problemática en común de las dos zonas de estudio residuos sólidos

La investigación presenta un enfoque metodológico basado en AcciónParticipación con interpretación cualitativa y cuantitativa, existe participación comunitaria, y un equipo de investigación, que con la ayuda de la comunidad planea estrategias pedagógicas para dar solución a la problemática identificada. Se resalta la participación de la administración del municipio de Baranoa, desde su competencia realizó aportes valiosos a este estudio. Otro aspecto relevante es el acompañamiento académico por parte del equipo de investigación "Educación Ambiental y desarrollo sustentable" de la Universidad del Atlántico.

Se puede concluir con esta investigación que se cumple uno de los criterios de la Educación Ambiental "Esta debe ser interinstitucional, intersectorial", ninguna institución, por si sola, puede abordar los problemas ambientales, proceso evidente al crear alianzas entre los habitantes del barrio Santa Ana, la administración Municipal y el equipo de investigación.

Palabras clave: Gestión Ambiental, Participación, ambiente, territorio, actores y agentes.

* Especialista en la Enseñanza de las Ciencias Naturales con énfasis en Educación Ambiental. Docente de la Escuela Normal Superior Santa Ana de Baranoa. Miembro del equipo de investigación. bosilra@hotmail.com. 
Abstract: The research project "Design and implementation of an environmental management system in Escuela Normal Superior Santa Ana and Santa Ana neighborhood in Baranoa town, that can promote the educative community participation in the appropriate use of solid waste" has been implemented since 2006 and currently it still prevails.

Through this project Teachers wanted to design and implement a manage environmental system in the school and in the neighborhood that promote the educative community and neighbors participation with the purpose of establishing alternatives solution about common problematic in two places in studies of solid waste.

The research presents a methodological approach based in action-participation with quality and quantity interpretation, besides there is community participation and a research group that together with the community plan pedagogic strategies to solve the identified problems. In the project it is important to stand out the participation of Baranoa government, which made valuable contributions to this research. Another relevant aspect is the academy accompaniment of the research group of Universidad del Atlántico, that is called "Environmental Education and Sustainable development".

From this research it can be concluded that one of the criteria of environmental education is fulfilled and it is necessary that it may be interinstitutional and intersectorial; any institution cannot approach itself the environmental problems, which is evident when alliances among Santa Ana neighbors, Baranoa government and the research group are created.

Key words: Environmental management, participation, environment, territory, actors and agents.

\section{INTRODUCCIÓN}

“La Educación Ambiental debe estar orientada hacia la formación para la participación en procesos de gestión; pues a través de ellos es que los individuos y los colectivos se hacen conscientes, tanto de sus competencias y responsabilidades para la toma de decisiones, en lo que a la problemas ambientales se refiere" (Educación Ambiental: Política Nacional, 2003,119p).

El Ministerio del Medio Ambiente y Desarrollo territorial, con la participación de diversos agentes sociales e instituciones involucrados en los problemas ambientales de la región, actualmente proponen el desarrollo de procesos de gestión a nivel nacional, regional y local para mejorar las condiciones de vida de la población y el desarrollo regional sostenible fortaleciendo la dimensión urbana, educativa y su relación con el entorno regional.

Desde esta perspectiva se justifica este proyecto de investigación "Diseño e implementación de un Sistema de Gestión Ambiental en la Escuela Normal Superior Santa Ana y el Barrio Santa del municipio de Baranoa que fomente la participación de la comunidad educativa en el manejo adecuado de los residuos sólidos", 
continuación me permito presentar esta experiencia significativa que se ésta llevando a cabo en el municipio de Baranoa desde el año 2006 y que se encuentra vigente, donde participan de forma activa Docentes de la Universidad del Atlántico, pertenecientes al equipo de Investigación, "Educación Ambiental y Desarrollo sustentable" y CIENSSA (Centro de Investigación de la Escuela Normal Superior Santa Ana).

Cabe señalar que uno de los propósitos de esta investigación es fomentar la participación de la comunidad educativa y del Barrio Santa Ana, a través del Sistema de Gestión Ambiental, el cual tiene una estructura organizacional que opera por fases en total cinco y que éste contempla el diseño e implementación del plan de manejo ambiental, donde se realizan una serie de acciones ambientales y pedagógicas propuestas por la comunidad educativa encaminadas a dar solución a la problemática ambiental que presenta la institución y el barrio Santa Ana.

La Escuela Normal Superior Santa Ana epicentro de esta investigación se encuentra situada en el municipio de Baranoa, ubicado éste en el noroccidente del Departamento del Atlántico, con una topografía de relieve plano y ligeramente ondulado; sus principales actividades económicas son: El comercio, la agricultura, la ganadería, la industria avícola, las confecciones, el transporte y pequeñas microempresas. Cuenta con servicios públicos como agua, luz, aseo, teléfono, Internet, transporte terrestre, canal local de televisión, dos emisoras, hospital, clínicas, Educación Preescolar, básica y media, con modalidades de bachillerato académico, comercial, industrial y pedagógico, además cuenta con Educación no formal y técnica. Hoy el municipio es considerado centro piloto de educación, música y cultura, tierra de paz, de bellas mujeres, prosperidad y alegría.

En este mismo sentido el barrio Santa Ana también objeto de estudio de ésta investigación, es un lugar relativamente joven levantado en terrenos correspondiente a la antigua cooperativa algodonera en el año de 1974, tiene 54 viviendas, cuenta con todos los servicios públicos, excepto sistema de alcantarillado ubicado aproximadamente a 300 metros de la Escuela Normal Superior Santa Ana.

\section{MATERIALES Y METODOS}

El proyecto se fundamenta en un enfoque metodológico basado en la acción participación con interpretación cuantitativa y cualitativa. El diseño acción participación está basado en la opinión y la conceptualización de la información obtenida. La esencia de la acción participación es la suma de evidencias que se obtienen de la realidad y los procesos reflexivos sobre los que se intenta darle sentido a esa realidad, este tipo de investigación constituye una variante de "la Investigación Acción Participación (IAP) donde existe participación comunitaria, pero generalmente hay un grupo gestor o agente externo que, con ayuda de la comunidad planea acciones y actividades para desarrollar el trabajo o proyecto comunitario; es siempre un proceso continuo en espiral, de acción-observaciónreflexión, propuesta de acción-realización de la misma"(Arnal, Del Rincón \& Latorre, 2003).

Los instrumentos de recolección de la información que permitieron identificar la problemática ambiental presente en las dos zonas de estudio son los siguientes: Cuestionarios semiestructurados, dirigidos a la comunidad educativa de la Escuela 
Normal Superior Santa Ana y a los habitantes del barrio Santa Ana; para el análisis de estos cuestionarios se emplearon categorías, que más adelante se esbozan. La unidad de análisis que se realiza es cualitativa, pero también se emplea la estadística descriptiva, se diseñan tablas y gráficos de las preguntas formuladas.

De igual forma para los obtención de los datos se realizaron entrevistas dirigidas a las autoridades del municipio de Baranoa, específicamente a las secretarias de despacho (De Salud, Educación, Planeación y Hacienda), con el objetivo de recopilar información relacionada con los problemas ambientales de Baranoa, del barrio Santa, la forma como es abordado el componente ambiental en el municipio, se verificó si existe un sistema de gestión ambiental en el municipio.

También fue necesario aplicar un formato para determinar los niveles de participación de los actores y agentes del sistema de gestión ambiental, este instrumento se aplicó al inicio del proyecto de investigación, posteriormente cuando se implementó el sistema de gestión ambiental. Este instrumento contiene unos indicadores de gestión que nos permiten detectar falencias y tomar correctivos, de igual forma nos proporciona una visión real sobre el aporte participativo de cada estamento, estudiante, padres de familia, docentes, directivos, habitantes del barrio Santa Ana.

Posteriormente se realizó la cartografía social de la Escuela y del barrio Santa Ana, este instrumento permitió la participación de los actores en las zonas de estudio, de igual forma facilitó la identificación de problemas ambientales y la formulación de alternativas de solución.

Se hizo una prueba piloto, en la escuela y el barrio Santa Ana, con el propósito de determinar la cantidad de residuos sólidos generados en los dos lugares; fue necesario este proceso porque al aplicar los cuestionarios semiestructurados y al realizar la cartografía social, estos instrumentos reflejaron este problema ambiental.

El equipo de investigación revisó el Plan Básico de Ordenamiento territorial de Baranoa (PBOT) y el Plan de Gestión Integral de Residuos Sólidos de este municipio (PGIRS), con el objeto de obtener una visión global y local de los problemas ambientales más críticos del municipio y del barrio Santa Ana.

Para orientar de forma más clara y precisa al lector, a continuación me permito explicitar la investigación con las siguientes fases:

\section{FASE I: PRELIMINAR}

En esta fase los lideres del proyecto, 44 jóvenes de la Escuela, son el semillero de la investigación, dinamizan el trabajo, aportan ideas, e interactúan con el equipo de investigación y los habitantes del barrio Santa Ana. El equipo de investigación son profesores de la Universidad del Atlántico y de la Escuela Normal, actúan como agentes académicos, asesores, pero también son participes de la investigación. Los lideres del proyecto, el equipo de investigación y los habitantes del barrio Santa Ana establecen 
relaciones reciprocas de observación, reflexión y acción, a través de mecanismos como el dialogo, negociación y concertación para buscar soluciones viables a la problemática ambiental; estos identifican actores y agentes que participan en el sistema de gestión ambiental, entendiendo actores como todas aquellas personas que ven afectada sus condiciones de vida, que influyen o reciben efectos propios de la contaminación ambiental, para este caso lo conforman la comunidad educativa de la Escuela Normal Superior Santa Ana y los habitantes del barrio Santa Ana del municipio de Baranoa.

Los agentes son los organismos públicos o privados, los representantes de grupos de poder, los empresarios, autoridades ambientales y en general aquellas instancias que van a participar de forma activa y que desde su competencia plantean alternativas de solución acorde con la problemática ambiental detectada.

Un aspecto fundamental para destacar es que en el año 2006 y 2007, tuvo participación activa la administración Municipal dirigida por el Doctor Carlos Zambrano Palacio, quien con su equipo de colaboradores, La Secretaria de Saneamiento Básico, planeación y Secretaria De Educación; hicieron aportes valiosos a éste proyecto, se pueden mencionar los siguientes: Se habilitaron espacios para que los jóvenes lideres de la investigación socializaran la fase diagnóstica del proyecto, La Secretaria de Planeación participó visitando el barrio Santa Ana, interactuando con algunos lideres de la comunidad de igual forma para conocer de cerca aspectos técnico ambientales inherentes al barrio en mención. De igual forma ésta Secretaria facilitó documentos Como el Plan Básico de Ordenamiento Territorial (PBOT) Y el Plan de gestión integral de residuos sólidos (PGIRS).

En este orden de ideas fue necesario socializar este año el proyecto de investigación a las autoridades del municipio de Baranoa, bajo la dirección del Doctor Clímaco Estrada Pérez, para este fin Los jóvenes lideres del proyecto coordinaron este proceso, en este evento participó de forma activa la Secretaria de Educación del Municipio, quien se comprometió a apoyar y financiar la publicación del Manual de Gestión Ambiental para las instituciones educativas, por considerar éste trabajo como la primera experiencia significativa que se está llevando a cabo en el municipio de Baranoa, además de sugerir que en lo posible el proyecto sirva de modelo para que otras instituciones educativas diseñen e implementen su Sistema de Gestión Ambiental. De igual forma la Secretaria de Salud ofrece sus servicios para liderar campañas en Pro de mejorar las condiciones de salud e higiene de los habitantes del barrio Santa Ana.

De esta manera se aprecia con claridad la participación activa y el grado de competencia de los actores y agentes, su intervención en el área de estudio. Seguidamente fue necesario que los lideres del proyecto, el equipo de investigación y los habitantes del barrio Santa Ana habilitaran espacios para concertar intereses acordes con la problemática ambiental detectada, plantearan alternativas de solución, sugiriendo mecanismos de control y evaluación. Dentro de los aspectos fundamentales para el éxito de éste proceso se destacan los siguientes:

- Es necesario reconocer por igual a todos los actores que participan en el proceso de gestión ambiental. 
- Recoger las opiniones de los actores sobre la problemática.

- Es importante confrontar las ideas de la comunidad del barrio Santa Ana con las ideas de los jóvenes líderes del proyecto y el grupo de investigación.

Además fue necesario que el grupo investigador y los líderes del proyecto estuvieran atentos a las siguientes funciones:

- Identificar a los genuinos representantes de los diversos grupos y sectores sociales, para el caso del barrio la junta de acción comunal, grupos religiosos, grupos de jóvenes organizados.

- Identificar las necesidades de todos los habitantes del barrio Santa Ana y determinar quienes están dispuestos a actuar en conjunto para solucionar los problemas que los afectan.

\section{FASE II: CARACTERIZACIÓN DEL AREA DE ESTUDIO}

El enfoque teórico de este proyecto se sustenta en el diseño e implementación de un Sistema de Gestión Ambiental, según la norma ISO 14001, este se define como una estructura organizacional donde se asumen responsabilidades, practicas, procedimientos, procesos y recursos para la implementación de la gestión ambiental.

Un concepto clave en el desarrollo de este sistema de gestión ambiental es la Participación, y esta se define como hacer parte de un todo, es contribuir, colaborar, cooperar, es invertir esfuerzos en torno a una meta u objetivo; es congregarse para un fin determinado, consiste en aunar esfuerzos voluntades frente a decisiones para buscar el bienestar colectivo y social.

Es fundamental señalar que el equipo de investigación, la comunidad educativa de la Escuela Normal Superior Santa Ana y los habitantes del barrio Santa Ana; priorizan, categorizan e identifican problemas ambientales; pero también planean y formulan estrategias pedagógicas y técnico ambientales que son el producto de la interacción, y concertación de las partes, este proceso o engranaje permite diseñar e implementar el plan de manejo ambiental educativo.

Tanto en la escuela como en otros espacios, la Educación Ambiental debe estar ligada a los problemas y potencialidades ambientales de las comunidades, ya que estos tocan de manera directa a los individuos y a los colectivos, están relacionados con su estructura social y cultural y es por medio de ellos, de la sensibilización y de la concientización de los mismos, que se puede incidir sobre las formas de actuar y de relacionarse con los diferentes componentes del entorno.

Otra reflexión del equipo que dirige este proyecto de investigación es que la escuela debe permitir la participación activa del niño y de toda la comunidad en la construcción del conocimiento, para encontrar alternativas de solución con la problemática ambiental particular.

Esta concepción debe ser acorde con el desarrollo propio de la comunidad a la que pertenece y de la sociedad de la cual hace parte, desde nuestras escuelas se puede 
propiciar la formación de agentes de cambio, multiplicadores conscientes de su papel trasformador dentro de una comunidad.

A continuación se referencian apartes de la fase II de la investigación, donde se tuvieron en cuenta los aspectos: Técnico- ambiental y el pedagógico.

- Técnico Ambiental: Se aplicaron los siguientes instrumentos de recolección de la información: Cuestionarios semiestructurados dirigidos a cada uno de los estamentos de la institución y a los habitantes del barrio Santa Ana, con el propósito de recolectar información acerca de la problemática ambiental de la escuela y el barrio, participación de la comunidad educativa en torno a los problemas ambientales, gestión ambiental Institucional, interinstitucionalidad y contextualización de la Educación Ambiental. Este instrumento fue analizado y se establecieron categorías, para determinar la problemática ambiental mas critica de la escuela y del barrio.

- Se realizaron entrevistas a las autoridades del municipio de Baranoa, con el objetivo de recopilar información relacionada con la problemática ambiental de Baranoa y del Barrio Santa Ana, participación de la administración para dar alternativas de solución en lo relacionado con problemática ambiental y además verificar si existe un sistema de gestión ambiental en el municipio.

- Se aplicó un formato para determinar los niveles de participación de los actores y de los agentes del sistema de gestión ambiental, éste se diligenció al inició del proyecto de investigación y durante la fase de implementación del proyecto, este permite develar las deficiencias en el proceso y plantear los correctivos necesarios.

- También se construyó la cartografía social de la Escuela Normal Superior Santa Ana y del barrio Santa Ana, esta estrategia metodológica estuvo encaminada a producir una visión sistémica del estado ambiental la escuela y del barrio. Se destaca la organización del trabajo, participaron docentes de las áreas de Tecnología, Humanidades, Sociales y Ciencias Naturales. Estos formaron equipos de trabajo con estudiantes de los diferentes grados de la escuela, padres de familia y el equipo de investigación, diligenciaron una guía de observación, realizaron recorridos dentro y fuera de la escuela y al momento de socializar su trabajo emplearon convenciones o dibujos para ser explícitos los problemas ambientales identificados.

Después se realizó una prueba piloto para determinar la cantidad de residuos sólidos generados en la escuela y el barrio Santa Ana. Se hizo en dos semanas, el pesaje se efectuó con una frecuencia interdiaria, atendiendo al horario de recolección de la empresa Aseo General S.A. que presta servicio en la Escuela y el barrio, esta actividad contó con la participación del personal de servicios generales de la escuela y padres de familia. En el barrio participaron habitantes de éste y el proceso fue liderado por el equipo de investigación y los jóvenes líderes del proyecto.

Finalmente se revisaron documentos del municipio de Baranoa, Plan Básico de Ordenamiento Territorial (PBOT) y Plan de Gestión Integral de Residuos Sólidos (PGIRS); 
es importante mencionar que el PBOT en su componente ambiental realiza la siguiente cita "Por desconocimiento y por tradición cultural, muchos de los habitantes de las fincas y de los alrededores arrojan basuras en cualquier sitio, en especial los arroyos, provocando contaminación por desechos orgánicos e inorgánicos, así mismo a nivel urbano no se tiene la conducta de reciclaje".

Pedagógico: En la Escuela Normal Superior Santa Ana se revisó el Proyecto Educativo Institucional (P.E.I), con el objetivo de identificar los problemas ambientales detectados en años anteriores específicamente desde el año 1982, donde desde esa fecha al presente se identifica con claridad la problemática ambiental de residuos sólidos. En torno a este problema se realizaron en diferentes épocas campañas, charlas, jornadas de aseo, pero se observa que aún persiste el problema. Otro aspecto que se indago en éste documento es el nivel de participación de las diferentes áreas del conocimiento en torno al problema, producto de éste análisis se determinó que la participación fue baja y que además los proyectos ambientales en su mayoría eran liderados o asumidos por docentes del área de Ciencias Naturales.

Además se construyó la historia del barrio Santa Ana, llevando registros o diarios de campo para este propósito los jóvenes que lideran el proyecto realizaron entrevistas a algunos habitantes del barrio, en especial, señores de la tercera edad para realizar una reconstrucción de la vida del barrio, ya que éste es relativamente nuevo. En esta parte de la investigación se acude a la tradición oral, porque a nivel del municipio no existe ningún documento que demuestre el legado del lugar.

Hay que destacar la participación de los jóvenes líderes del proyecto de investigación, 44 estudiantes actualmente cursan $11^{\circ}$. Ellos se constituyen en el semillero de este proyecto pues ingresaron a éste desde el año 2006. Otro aspecto relevante es que algunos son habitantes del barrio Santa Ana; es fundamental mencionar la dinámica de trabajo que emplea el grupo, están organizados por equipos de trabajo, el de logística, ecológico y de difusión; cada equipo interactúa en torno a una misma acción o estrategia que se esté llevando a cabo en el proyecto.

\section{FASE III: DIAGNOSTICO}

La tercera fase corresponde a la elaboración del diagnostico, teniendo en cuenta la información recogida en la fase anterior, se hizo el análisis del aspecto técnico-ambiental y el pedagógico, la información se analiza cualitativa y cuantitativamente, se definieron categorías que facilitaron determinar la problemática ambiental que presenta la institución y el barrio Santa del municipio de Baranoa. Ésta fase también sirvió para que los actores del sistema de gestión ambiental identificaran con claridad los problemas ambientales que los afectan, en este sentido se analizan las causas y efectos de cada problema, se clasifican y se categorizan desde diferentes perspectivas, es decir jerarquizarlos y analizar los procesos históricos que llevaron a la situación actual y proyectarlos a futuro.

En la primera interacción de los actores, agentes y grupo de investigación se desarrolla un proceso de observación y reconocimiento de la situación actual y la posible situación 
futura del área (Barrio Santa y Escuela Normal Superior Santa Ana). Esta fase nos permite elaborar un diagnóstico real.

\section{RESULTADOS Y DISCUSIÓN}

En esta investigación la problemática ambiental que se identificó es que los niveles de participación de los actores y agentes son mínimos en lo relacionado con la resolución problemas ambientales, para apreciar el aporte participativo de cada estamento (Estudiantes, docentes, directivos, padres de familia y habitantes del barrio Santa Ana) es importante comparar su comportamiento frente al conjunto de la comunidad educativa. "Para este fin los totales horizontales de la siguiente tabla, se llevan a un pentágono dada la evaluación de cinco estamentos, el pentágono tiene áreas sombreadas según el grado de participación" (Sepúlveda L; Tobasura I, 1997,102 p)

El área definida entre los valores 0 y 5 se considera de NO PARTICIPACION

El área definida entre los valores mayores de 5 y menores o iguales a 15 se considera de PARTICIPACION EN ESPACIOS DE DIALOGO.

El área definida entre los valores mayores de 15 y menores o iguales a 20 se considera de PARTICIPACION EN FASE DE PODER COMPARTIDO.

El estudio de éste gráfico permitió identificar los estamentos que requieren mayor apoyo para mejorar su proceso de participación en la gestión del proyecto. Claramente se aprecia que el estamento padres, habitantes del barrio y estudiantes presenta niveles bajos que corresponde a Participación en espacios de dialogo. El pentágono en color rojo circunscrito dentro del ideal corresponde al estudio inicial de la investigación, es decir antes de implementar el Sistema de Gestión Ambiental. (Ver figura 1).

\section{CUADRO RESUMEN DE LA EVALUACION DE LA PARTICIPACION POR ESTAMENTO Y PARAMETRO. ANTES DE IMPLEMENTAR EL SISTEMA DE GESTION AMBIENTAL.}

\begin{tabular}{|c|c|c|c|c|c|c|}
\hline Parámetro & $\begin{array}{c}\text { Definición de } \\
\text { problemas }\end{array}$ & Liderazgo & Organización & $\begin{array}{l}\text { Movilización de } \\
\text { recursos }\end{array}$ & $\begin{array}{c}\text { Compromiso } \\
\text { acciones }\end{array}$ & Totales \\
\hline Estudiantes & 2 & 2 & 2 & 1 & 2 & 9 \\
\hline Docentes & 2 & 2 & 3 & 1 & 2 & 10 \\
\hline Directivos & 2 & 2 & 3 & 1 & 1 & 9 \\
\hline Padres & 2 & 2 & 2 & 0 & 1 & 7 \\
\hline
\end{tabular}




\begin{tabular}{|c|c|c|c|c|c|c|}
\hline $\begin{array}{c}\text { Habitantes } \\
\text { Del barrio }\end{array}$ & 2 & 2 & 2 & 1 & 1 & 8 \\
\hline Totales & $\mathbf{1 0}$ & $\mathbf{1 0}$ & $\mathbf{1 2}$ & $\mathbf{4}$ & 7 & 43 \\
\hline
\end{tabular}

Figura 1. Evaluación de la participación. Antes de implementar el Sistema de Gestión Ambiental.
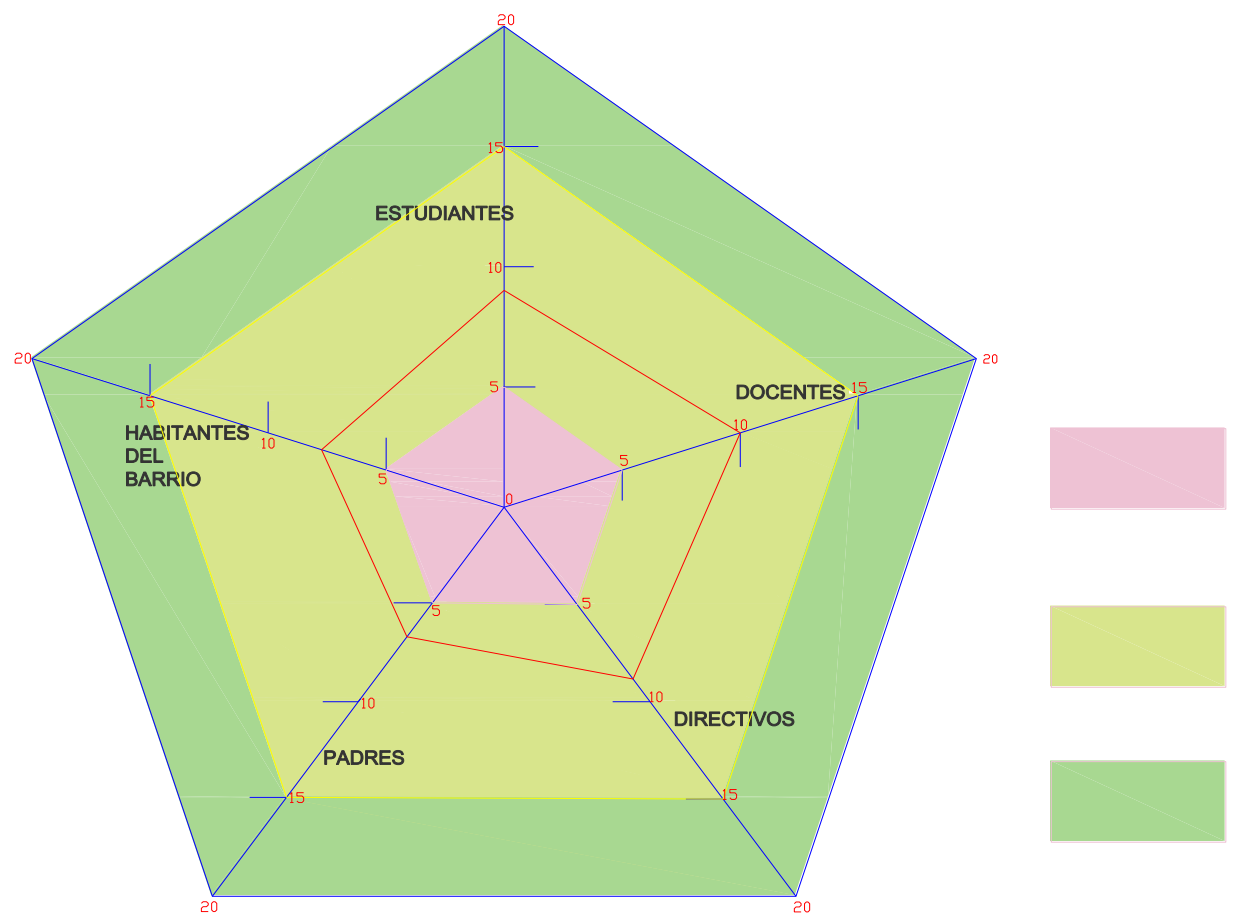

NO PARTICIPACION

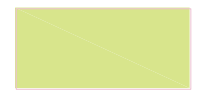

PARTICIPACION EN FASE DE DIALOGO

PARTICIPACION REAL

También destaco algunas de las preguntas relevantes en esta investigación y que se encuentran inmersas en los cuestionarios semiestructurados que se les aplicaron a la comunidad educativa y a los habitantes del barrio Santa Ana. Llama la atención como los cuestionados expresan que es mala la continuidad de las acciones ambientales desarrolladas en la escuela y el barrio Santa Ana, un 53\% así lo ratifica; también expresan que los residuos sólidos es el problema más critico que afecta a las dos zonas de estudio, el $47 \%$ lo evidencia. (Ver gráficos № 1 y 2). 


\section{Gráfico 1. Continuidad de las Acciones Ambientales (Escuela/Barrio Santa Ana)}

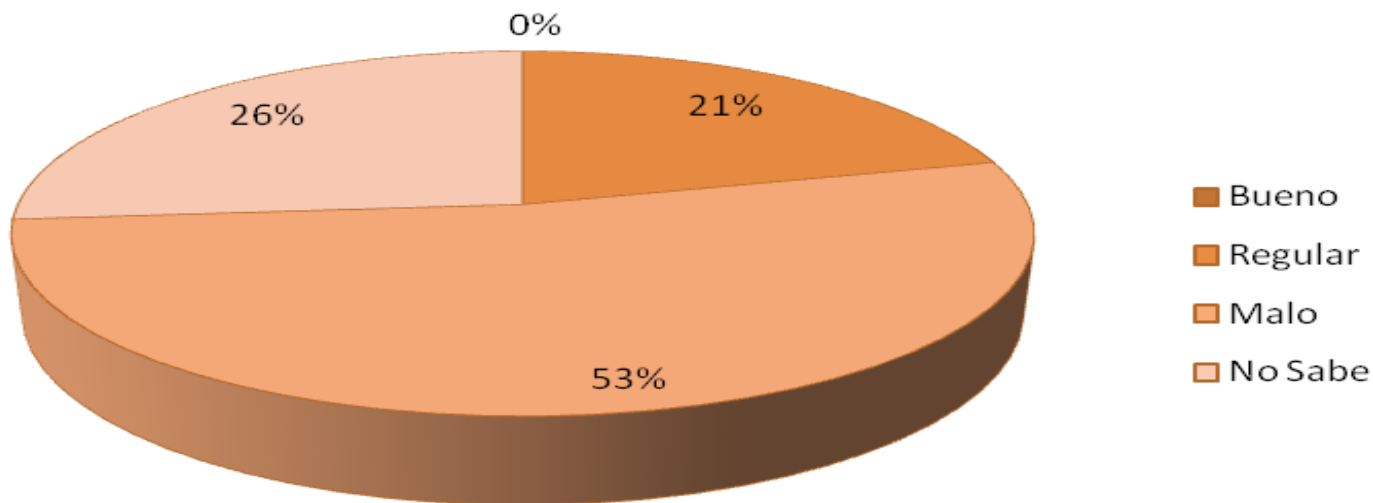

Gráfico 2. ¿De los siguientes problemas ambientales, cuál consideras debe ser abordado por el Proyecto de investigación?

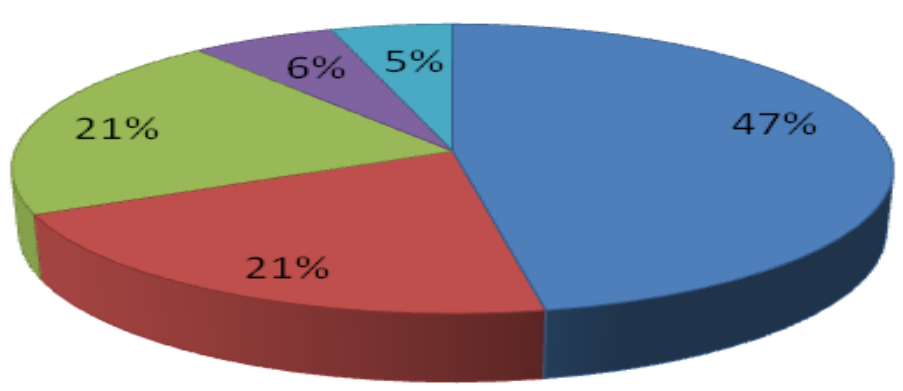

Residuos Sólidos

Deforestación

Contaminación Sonora

Contaminación por Ag Negras

$\square$ Otros

Otro aspecto a tener en cuenta en el análisis es que al realizar la revisión del Proyecto Educativo Institucional de la Escuela Normal Superior Santa Ana, es claro que no existe un sistema de gestión ambiental que oriente la identificación de problemas ambientales y proponga alternativas de solución. En este sentido cuando se realizó la entrevista a las autoridades del municipio de Baranoa también dejaron claro que no existe un sistema de gestión ambiental local o estructura orgánica que regule las acciones de protección y conservación del ambiente.

Sin duda alguna es necesario que el grupo investigador y los líderes del proyecto diseñen el Sistema de Gestión Ambiental, pero no se comprometen con la implementación de soluciones que no están a su alcance, porque esto puede contribuir a perder credibilidad en la comunidad. Es importante que la comunidad delimite y clasifique los ámbitos o 
territorio dentro de los cuales se pretende solucionar los problemas identificados. Los más importantes son: El ámbito geográfico o natural, el ámbito económico, el social, el político administrativo y el ámbito institucional o funcional.

Las propuestas de solución deben ser realistas y por lo tanto hay que determinar como mínimo lo siguiente:

- Dónde se tomaran las medidas pertinentes.

- Que otras alternativas de solución existen.

- Que instituciones o personas estarán involucradas en la adopción de medidas.

- Que insumos supone cada alternativa.

En este proyecto de investigación se observó en la figura 1, que el pentágono al inicio de la investigación es irregular y está inscrito dentro del ideal; pero a medida que se avanza en la investigación y se implementa el sistema de gestión se vuelve a aplicar el instrumento "Pentágono de Participación" se identifica con claridad que se amplía progresivamente, según la Figura 2, quiere decir esto que las estrategias pedagógicas o acciones empleadas apuntan a mejorar los parámetros y a subir los niveles de participación.

\section{CUADRO RESUMEN DE LA EVALUACION DE LA PARTICIPACION POR ESTAMENTO Y PARAMETRO. (DURANTE LA IMPLEMENTACION DEL SISTEMA DE GESTION AMBIENTAL)}

\begin{tabular}{|c|c|c|c|c|c|c|}
\hline Parámetro & $\begin{array}{l}\text { Definició } \\
\text { n de } \\
\text { problema } \\
\text { s }\end{array}$ & $\begin{array}{l}\text { Lideraz } \\
\text { go }\end{array}$ & $\begin{array}{l}\text { Organizaci } \\
\text { ón }\end{array}$ & $\begin{array}{c}\text { Movilizació } \\
\text { n de } \\
\text { recursos }\end{array}$ & $\begin{array}{l}\text { Compromi } \\
\text { so } \\
\text { acciones }\end{array}$ & Totales \\
\hline Estudiantes & 4 & 4 & 4 & 3 & 3 & 18 \\
\hline Docentes & 3 & 3 & 3 & 2 & 3 & 14 \\
\hline Directivos & 3 & 3 & 4 & 2 & 2 & 14 \\
\hline Padres & 4 & 2 & 4 & 1 & 2 & 13 \\
\hline $\begin{array}{c}\text { Habitantes } \\
\text { del barrio }\end{array}$ & 3 & 4 & 4 & 3 & 4 & 18 \\
\hline Totales & 17 & 16 & 19 & 11 & 14 & 77 \\
\hline
\end{tabular}




\section{Figura 2. Evaluación de la participación. Durante la implementación del Sistema de Gestión Ambiental.}
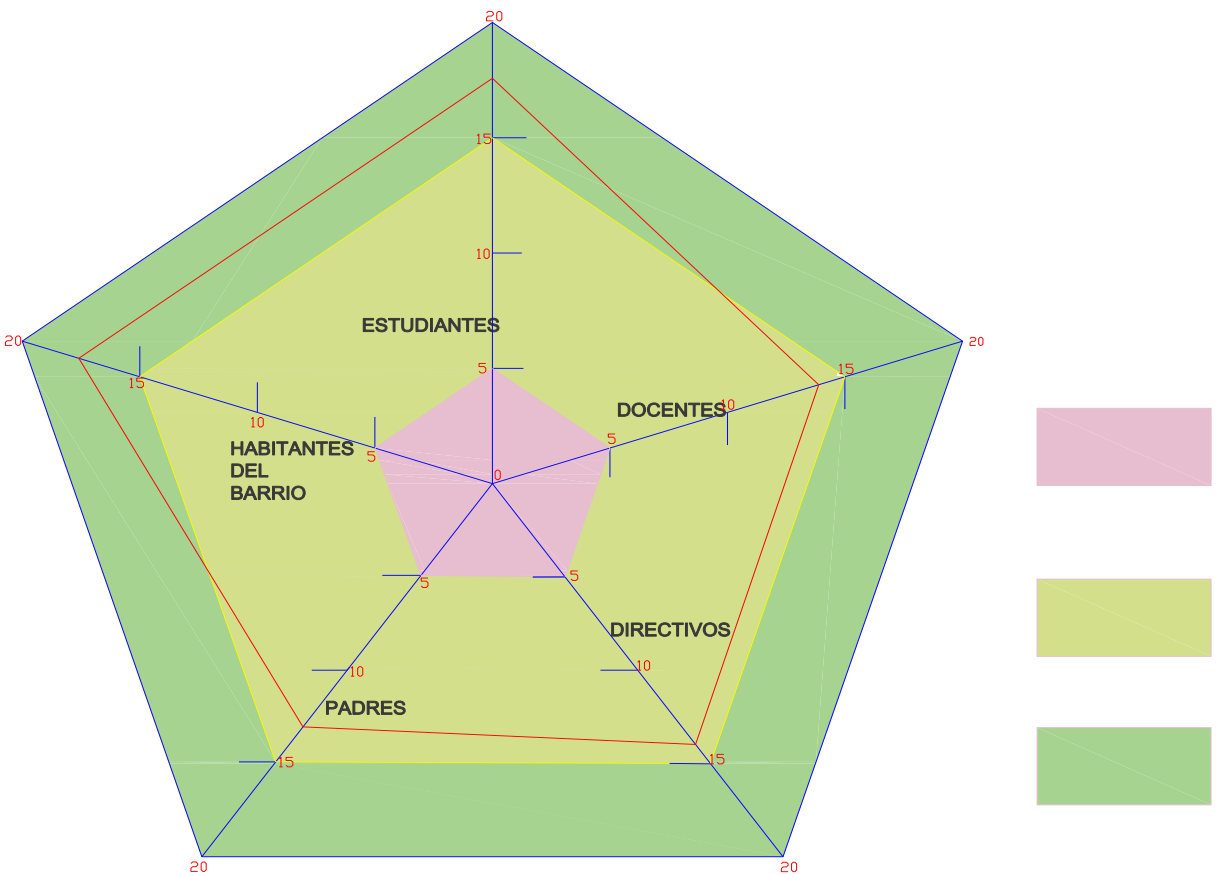

NO PARTICIPACION

PARTICIPACION EN FASE DE DIALOGO

PARTICIPACION REAL

Son tantos los problemas como alternativas de solución que pueden presentarse en el área de estudio, en el caso del barrio Santa Ana, la problemática de residuos sólidos no es reciente, de acuerdo a lo manifestado por sus habitantes el hecho de que el barrio este rodeado por arroyo grande, microcuenca del municipio, convierte el lugar en área vulnerable para este tipo de problema ambiental. Sin embargo a través de la Junta de Acción Comunal se han impetrado acciones tendientes a solucionar el problema. Es evidente que los lideres del proyecto y el equipo de investigación consideraron fundamental la vinculación de grupos $\mathrm{u}$ organizaciones que planearan y ejecutaran diferentes alternativas de solución. $\mathrm{Y}$ aún más es necesario la intervención de las autoridades municipales para que desde su competencia regulen y permitan cumplir normas inherentes a éste problema.

Es menester en éste tipo de trabajo, también hacer énfasis en que toda actividad educativa en materia de ambiente, debe tender a la formación de la responsabilidad individual y colectiva, buscar un compromiso real del individuo con el manejo de su entorno inmediato, teniendo en cuenta referentes locales y globales. Desde esta perspectiva se puede afirmar que el individuo puede reconocerse y reconocer a los demás dentro de unos criterios claros de diversidad, comprender la dinámica social y sus elementos de evolución, valorando su cultura y su mundo. A su vez "le permite a los ciudadanos ser críticos y participes en cualquier proceso de gestión propiciando una verdadera formación en la responsabilidad".(Bayona M, 2002,41 p) 


\section{FASE IV: PLAN DE MANEJO AMBIENTAL EDUCATIVO}

El plan de acción ambiental consiste en una estructura organizacional donde se realizan una serie de acciones ambientales y pedagógicas propuestas por la comunidad educativa encaminadas a dar solución a la problemática que presenta la institución y el barrio Santa Ana.

Es necesario mencionar que "El plan de manejo ambiental educativo plantea una serie de estrategias pedagógicas y técnico ambientales tendientes a permitir la participación activa de la comunidad educativa" (Pedraza N, N, 2002,209p).

Se realizan acciones con la comunidad educativa, con los habitantes del barrio Santa Ana y las autoridades del municipio de Baranoa, específicamente las Secretarias De Educación, Hacienda y de Salud; cada una desde su competencia plantea alternativas de solución acordes con la problemática identificada.

Por lo tanto, la idea de gestión y el actuar responsable son en ultimas garantes del sistema de gestión ambiental.

\section{FASE V: EJECUCION DEL PLAN DE MANEJO AMBIENTAL EDUCATIVO}

Avanzando en las fases encontramos la ejecución del plan de manejo ambiental educativo en el cual las acciones o estrategias pedagógicas que se realizan presentan diversas escalas: Corto, mediano y largo plazo, según el tipo de acción que se este llevando a cabo. Cabe destacar que en el desarrollo de estas acciones participan actores y agentes del sistema de gestión ambiental. A continuación se referencian algunas:

- Tour en el barrio Santa Ana y la escuela, para realizar observaciones directas e identificar problemas ambientales.

- Consolidación de los guardianes ambientales de la Escuela Normal Superior Santa Ana de Baranoa, para trabajar sobre el proceso de separación y clasificación de los residuos sólidos.

- Foro "Manejo adecuado de residuos sólidos", Taller "Impacto de los desechos sólidos sobre la salud", dirigido a la escuela y el barrio Santa Ana.

- Difusión del proyecto de investigación y la problemática identificada a través de la emisora comunitaria "Fiesta Stereo"

- Concurso en la escuela, para mejorar las condiciones ambientales de ciertos lugares impactados por residuos sólidos

- Diseño de retablos con mensajes y dibujos ambientales para realizar labor pedagógica de sensibilización a los actores y agentes del Sistema.

- Patrocinio de la empresa privada TERPEL del norte, se vincula al proyecto donando canecas para realizar la separación de los residuos sólidos generados en la escuela y el barrio.

- Consolidación del semillero de Gestión Ambiental adscrito a la Universidad del Atlántico, se vinculan los 44 jóvenes lideres de éste proyecto. 
- Apoyo económico por parte de las autoridades municipales para realizar la publicación del "Manual de Gestión Ambiental para las Instituciones Educativas" con el objetivo de orientar a las escuelas y colegios del municipio de Baranoa y del Departamento del Atlántico para que diseñen e implementen su Sistema de Gestión Ambiental.

Es importante considerar que construir una concepción de manejo adecuado del ambiente en el marco de un desarrollo sostenible, que este de acuerdo con las necesidades y aspiraciones de las poblaciones, en sus contextos particulares, requiere el trabajo correspondiente de un concepto de gestión.

Otro aspecto relevante dentro de este proceso es que tanto actores como agentes deben tener claro que los problemas ambientales no pueden tratarse exclusivamente desde su dimensión natural, fisicoquímica y biológica. Para su comprensión es indispensable considerar simultáneamente su dimensión humana, es decir, tener en cuenta las implicaciones demográficas, sociales, económicas, técnicas, políticas y culturales.

A continuación se resume la investigación a través del siguiente flujograma: 
DISEÑO E IMPLEMENTACION DE UN SISTEMA DE GESTION AMBIENTAL EN LA ESCUELA NORMAL SUPERIOR SANTA ANA Y EL BARRIO SANTA ANA, QUE FOMENTE LA PARTICIPACION DE LA COMUNIDAD EN TORNO A LA PROBLEMÁTICA RESIDUOS SÓLIDOS

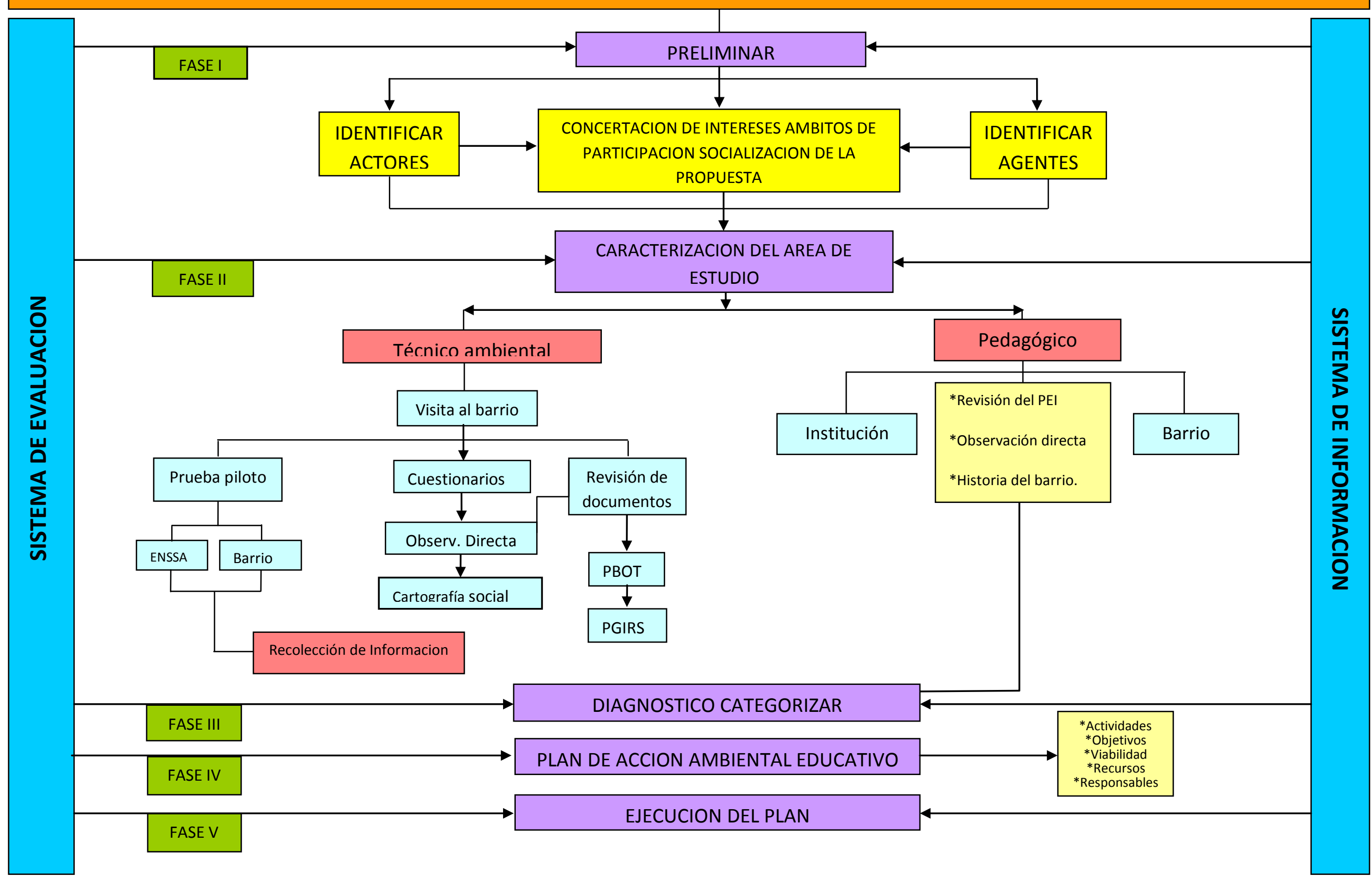




\section{SISTEMA DE INFORMACION Y EVALUACION PERMANENTE}

Durante el desarrollo de cada una de las fases del sistema de gestión, se llevó a cabo un sistema de evaluación, seguimiento e información permanente, que consistía en la realización y revisión de cada uno de los aspectos inherentes a cada fase, con el fin de identificar fortalezas y debilidades para aplicar correctivos en la fase siguiente. Para ejercer un mejor control sobre el sistema de gestión además de manejar las escalas a corto, mediano y largo plazo se emplean indicadores de gestión, para efectos de un seguimiento y monitoreo de las acciones o estrategias pedagógicas que van a permitir solucionar los problemas detectados.

No se puede orientar un proceso de participación comunitaria sin que se ejerza un sistema de evaluación que permita hacer ajustes, es por esto que el control permanente tiene que ser coherente con la formulación de estrategias pedagógicas que se seleccionaron. Participación aquí significa intervención en la toma de decisiones, el control y evaluación permanente de cada fase o proceso.

\section{CONCLUSIONES}

A continuación se presentan las conclusiones que se derivan de esta investigación:

- Para el éxito de un Sistema de Gestión Ambiental se requiere fomentar los niveles de participación, como los espacios de dialogo y poder compartido, haciendo uso de mecanismos como la negociación y concertación para llegar a acuerdos por consenso entre la Escuela Normal y el barrio Santa Ana en aras de buscar solución a la problemática ambiental.

- Se fortaleció la integración de la escuela con la comunidad, es importante esta relación porque a través de ésta se generan procesos de transformación que inciden en el desarrollo individual y comunitario. Este tipo de proyecto no puede construirse por fuera de un proceso formativo íntimamente relacionado con la familia, la escuela y todos aquellos que de una u otra manera hacen parte de la comunidad.

- “El manual de Gestión Ambiental para las Instituciones Educativas, experiencia ENSSA", publicación con registro ISBN puede considerarse como una guía $o$ herramienta que oriente la formulación y resolución de problemas ambientales en las instituciones educativas del Departamento del Atlántico. A través de este manual se pretende orientar a profesores, estudiantes, padres de familia, autoridades administrativas y ambientales a planear, sistematizar, organizar y ejecutar acciones organizadas tendientes a dar solución a los problemas ambientales de su entorno. Está organizado en una fundamentación teórica, legal y ambiental donde se describen las fases que componen el Sistema de Gestión Ambiental y una segunda parte la constituye la experiencia de la Escuela Normal Superior Santa Ana en el diseño e implementación del Sistema de Gestión Ambiental. Actualmente el Manual de Gestión Ambiental está implementado en la Escuela. 
- Las estrategias pedagógicas como mediación y fortaleza para mejorar los niveles de participación, es claro que actores y agentes, dinamizan su trabajo, concertan acciones y son más comprometidos con la problemática en común de las dos zonas de estudio.

- Es importante considerar que construir una concepción de manejo adecuado del ambiente en el marco de un desarrollo sostenible, que este de acuerdo con las necesidades y aspiraciones de las poblaciones, en sus contextos particulares, requiere el trabajo correspondiente de un concepto de gestión. Otro aspecto relevante dentro de este proceso es que tanto actores como agentes deben tener claro que los problemas ambientales no pueden tratarse exclusivamente desde su dimensión natural, fisicoquímica y biológica. Para su comprensión es indispensable considerar simultáneamente su dimensión humana, es decir, tener en cuenta las implicaciones demográficas, sociales, económicas, técnicas, políticas y culturales.

- “El componente de participación comunitaria, como una acción ciudadana tendiente a resolver las necesidades en un grupo de personas que habita un territorio", tienen una vida en común, pueden compartir costumbres, creencias y tradiciones semejantes, los cuales generan un sentido de pertenencia y una dinámica de acuerdo a los problemas y recursos a trabajar para mejorar la calidad de vida de la comunidad. (Londoño B, 1998, $34 \mathrm{p})$.

- Finalmente se puede decir que a través de ésta investigación se logró alcanzar uno de los criterios que se manejan en la Educación Ambiental "Esta debe ser interinstitucional e intersectorial, ninguna institución, por si sola, puede abordar los problemas ambientales", proceso fue evidente al establecer alianzas entre la Junta de Acción Comunal del Barrio Santa Ana, el equipo de investigación y la administración municipal de Baranoa, para la resolución de la problemática ambiental a pesar de los cambios de administración por finalización de periodo.

\section{Bibliografía}

- ARNAL J; DEL RINCON D; LATORRE A 2003. Bases metodologicas de la investigación educativa.(1ra Ed.) Ediciones experiencia. España. 315 p.

- Educación Ambiental: Política Nacional. Bogotá. 2003. 119 p.

- Plan Básico de Ordenamiento Territorial [PBOT]: Concejo municipal de Baranoa, 2003.111 p

- SEPULVEDA L; TOBASURA I. 1997. Proyectos Ambientales Escolares: Estrategia para la formación ambiental. Cooperativa editorial Magisterio. Santa Fe de Bogotá. 102 p.

- BAYONA M. 2002. Ciudadanía y construcción de lo público. Serie “Construyendo región". Módulo. Magdalena Medio. 41 p.

- LONDOÑO B. 1998. Manual nuevos instrumentos de participación ambiental. Consultoría ambiental y colectiva. Bogotá. 34 p.

- PEDRAZA N, N. 2002. Plan de acción para formadores ambientales. Editorial Magisterio. Bogotá. 209 p. 